\title{
Interatrial Block and Intermittent Paroxysmal Atrial Fibrillation in Amyotrophic Lateral Sclerosis Patient Treated with Flecainide
}

\author{
Athanassios Antonopoulos, MD, PhD, FESC ${ }^{*}$ iD and Giulia Ricci Lucchi, MD \\ Cardiology Clinic, Ospedale per gli Infermi, Faenza, AUSL Romagna, Faenza (RA), Italy \\ *Corresponding author: Athanassios Antonopoulos, MD, PhD, FESC, UO Cardiologia, Cardiology Clinic, \\ Ospedale per gli Infermi, Viale Stradone 9, 48018 Faenza (RA), Italy, Tel: 0546/601238, Fax: 0546/601110
}

\author{
Keywords \\ Interatrial block, Lateral amyotrophic sclerosis, Atrial fibril- \\ lation, Flecainide
}

\section{Introduction}

Amyotrophic lateral sclerosis (ALS) is a neurodegenerative disorder affecting both upper and lower motor neurons. Cardiovascular consequences related to ALS are relatively under appreciated. Autonomic dysfunction in ALS is common. In fact, patients with ALS often exhibit bulbar dysfunction impacting centrally mediated vagal and sympathetic nerves with resultant greater autonomic dysfunction. The consequences of disruptions in the autonomic reflex loop on the cardiovascular system include chronotropic incompetence and vasomotor instability [1-3]. Arrhythmias associated with ALS may occur as a result of autonomic dysfunction. Bradycardia likewise can be explained in ALS by sympathetic denervation followed by the predominance of vagal function. Autonomic dysfunction begins with vagal release with enhanced sympathetic tone causing tachycardia. As patients later progress with ALS, sympathetic denervation occurs, followed by vagal predominance, leading to the development of bradycardia. In a study examining the effects of vagal tone on the sinoatrial node and AV node, in patients with a history of syncope, sinus arrest or bradycardia, it was found that increased vagal tone contributed to prolongation in the sinus cycle length [4]. The above may be the expression of the presence of denervation edema. In fact, in a previous study with cardiac magnetic resonance (CMR) was observed an early gadolinium enhancement which in a disease with denervation, as the main muscular pathology, is likely to be associated with denervation edema [5].

Interatrial block (IAB) is a frequent condition in old age, reaching $40 \%$ in the over $70+$ year-old individuals $[6,7]$. Inter-atrial block is strongly associated with supraventricular arrhythmias, particularly with AF in many clinical contexts. Furthermore, IAB increases the risk of stroke [7] and appears to be associated with dementia.

The association of IAB with the incidence of AF was confirmed in different contexts [8-10]. We describe here a case of a patient with ALS in whom detection of advanced IAB during either on resting EKG or in a Holter registration was associated with intermittent paroxysmal atrial fibrillation episodes.

To our knowledge this is the first reported case with interatrial block and intermittent AF in a Holter registration in a patient with ALS.

\section{Case Presentation}

A 70-year-old Caucasian men with a 5 year history of ALS was admitted to the hospital following worsening of an unsteady gait, vertigo, nausea, and vomiting. The patient was hypertensive under treatment. Moreover, no chest pain was reported during the last 3 months. He has experienced dyspneic episodes and intermittent use of a CPAP for breath amelioration. One year ago he underwent a cardiac ultrasound that demonstrated normal biventricular systolic function: He had degeneration of the mitral and aortic valves with no transvalvular gradients. There was left atrial dilatation 


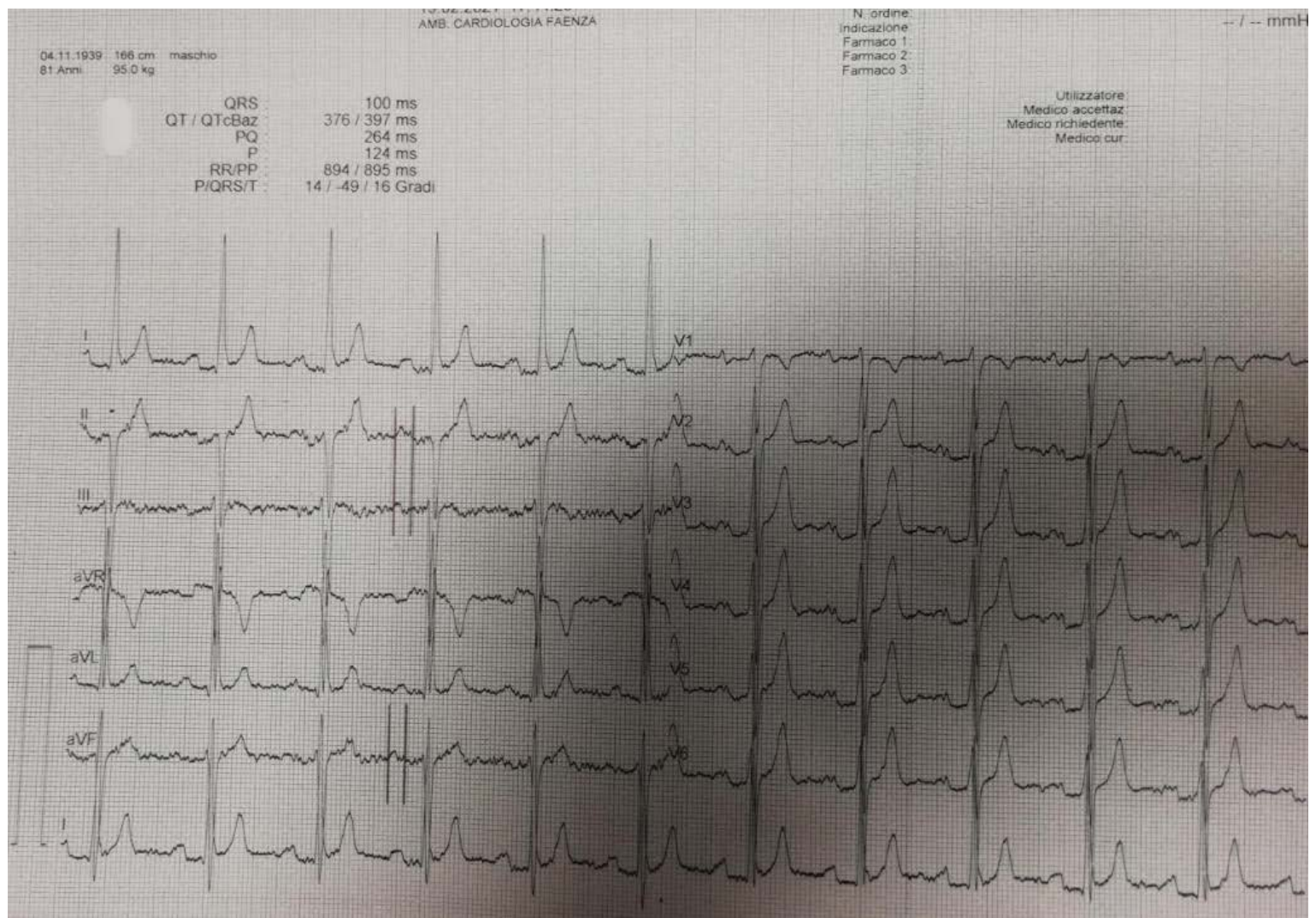

Figure 1: ECG: 12-lead ECG (with $40 \mathrm{~mm} / \mathrm{mV}$ magnification and $25 \mathrm{~mm} / \mathrm{s}$ paper velocity) at rest in the room. ECG shows sinus rhythm. P wave duration is increased (124 ms), P waves have a biphasic morphology in leads III and aVF, and a bimodal morphology in lead II, consistent with advanced IAB.

and no pericardial effusion. In the last 2 months that patient was under treatment with ibersartan $150 \mathrm{mg}$ daily, atorvastatin $20 \mathrm{mg}$ one time daily and hydroclorothizide $25 \mathrm{mg}$ once daily. Upon admission a chest X-ray demonstrated clear lung fields. His arterial blood gas revealed a $\mathrm{PH} 7.4$ with $\mathrm{PO}_{2} 77 \mathrm{mmHg}$ and $\mathrm{pCO}_{2} 38 \mathrm{mmHg}$. On neurologic examination, the patient had appropriate and fluent speech with no evidence of dysartria. Mental status examination was normal. There was normal upper and lower extremity strength. Deep tendon reflexes were normal and symmetric. His gait demonstrated severe ataxia. The patient's initial laboratory evaluation was unremarkable. Results of complete cell count, electrolytes and liver functions tests were within normal limits. Shortly after the initial evaluation the patient complained of a sudden onset of shortness of breath and palpitations. An initial EKG showed a sinus tachycardia $102 \mathrm{bpm}$. A pulmonary embolism was suspected. A pulmonary angio-TC was performed and was found to be negative. Additional laboratory analysis revealed normal thyroid function test results and C-reactive protein. Computed tomography of the head was normal. A bedside echocardiogram demonstrated normal cardiac chamber sizes with normal valvular function and contractility. Pericardial space was normal. In the department room a new resting EKG was obtained. A careful observation of the $P$ wave morphology for duration and voltage in a resting EKG (Figure 1) which when magnified at $40 \mathrm{~mm} / \mathrm{mV}$ and $25 \mathrm{~mm} / \mathrm{s}$ paper velocity revealed an advanced interatrial block alteration (duration 124 $\mathrm{ms}$, and the polarity was biphasic in leads II, III, aVF). Moreover, a holter registration (Figure 2 and Figure 3) was obtained revealing frequent intermittent episodes of paroxysmal atrial fibrillation. Moreover, a different duration of $\mathrm{P}$ wave was observed on the Holter measurement. Furthermore, during his hospitalization the patient received high dose methylprednisolone $(1 \mathrm{gr} /$ die) with gradual improvement in his neurologic abnormalities. Moreover, a prophylactic antiarrhythmic therapy with flecainide $100 \mathrm{mg} 2$ times daily for 5 days followed by $50 \mathrm{mg}$ two times daily for the next period. Additionally, based on patient CHA2DS2VASc score equal to 4 , apixaban $5 \mathrm{mg}$ twice daily was also prescribed. Another holter EKG monitoring was performed 20 days later revealing no paroxysmal atrial fibrillation episodes.

\section{Discussion}

It is well accepted that IAB has been described to predict occurrence of newly onset and recurrent episodes of atrial fibrillation [6]. The prevalence of advanced and partial IAB is $1 \%$ and $9.7 \%$ respectively, in the general population and they are both associated with increased risk of $\mathrm{AF}$ [7]. Its prevalence increases with age, reaching a prevalence of $26 \%$ of advanced $I A B$ and $20.1 \%$ of partial IAB in individuals $>100$ years of age [11]. Aging is associated with a progressive increase in the degree of atrial fibrosis and modification in the cardiac conduction system, which can be associated with IAB. AF, atri- 


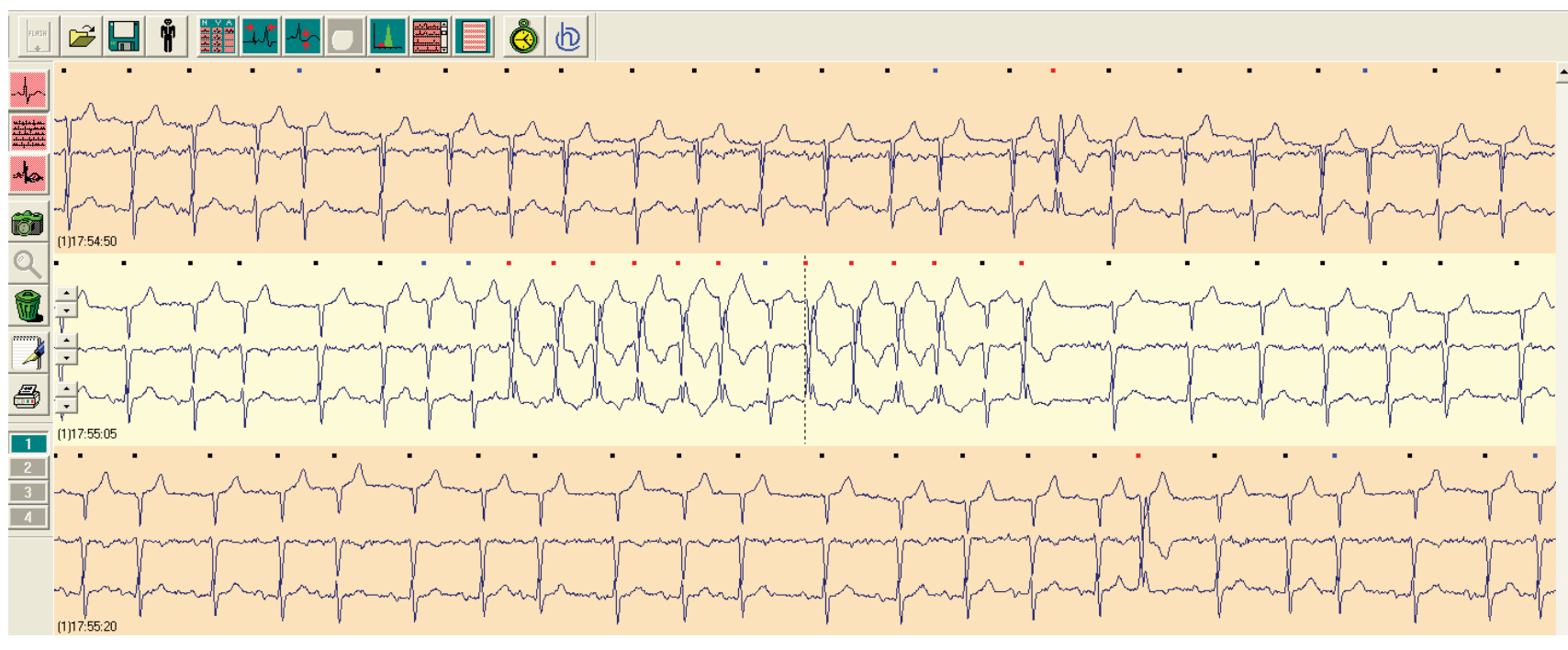

Figure 2: A short paroxysmal atrial fibrillation event on Holter EKG monitoring.

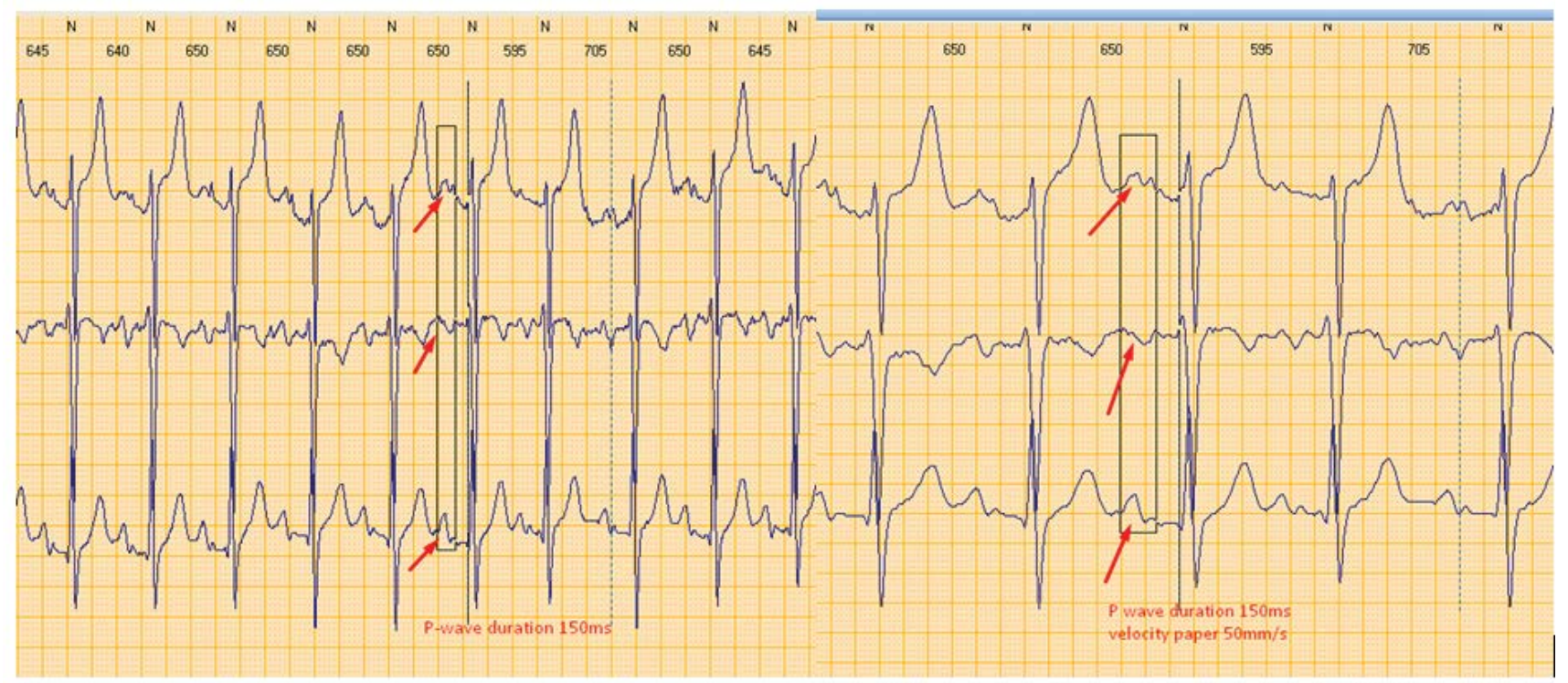

Figure 3: Interatrial block as it has been shown on Holter EKG monitoring. $P$ wave measurements are obtained with semiautomatic application of Holter software at different velocity paper $25 \mathrm{~mm} / \mathrm{s}$ (left part) and $50 \mathrm{~mm} / \mathrm{s}$ (right part) in order to discriminate better $\mathrm{P}$ wave morphology.

al premature beats and runs of atrial arrhythmias are also associated with age and with IAB [12]. On the other hand, previous studies have reported that the association between ALS, a demyelinating disease of the brain stem, and abnormalities of cardiac conduction exists. Autonomic dysfunction begins with vagal release with enhanced sympathetic tone causing tachycardia. As patients later progress in ALS, sympathetic denervation occurs, followed by vagal predominance, leading to the development of bradycardia [13].

In a study examining the effects of vagal tone on the sinoatrial node and AV node, in patients with a history of syncope demonstrate sinus arrest or bradycardia. Increased vagal tone contributed to prolongation in the sinus cycle length [4]. The last finding may be caused by the presence of denervation edema. In our case an edema extension across Bachman branches may induce a conduction alteration process, resulting in IAB. In fact, a previous study involving CMR observed early gadolinium enhancement which in a disease with denervation, as the main muscular pathology, is likely to be associated with denervation edema [5]. Previous studies have shown that abnormalities in CMR were frequent in ALS, in particular the early gadolinium enhancement $(77 \%)$ as a parameter of enhanced extracellular volume, that is either capillary leakage or fibrosis. The extension of that is difficult to define. Additionally, slowing of the sinoatrial node and inhomogeneous shortening of atrial refractory periods due to vagal stimulation in conjunction with fibrosis can facilitate IAB and develop AF. Another previous observation, using CMR and late gadolinium enhancement of the upper part of the septum, involving Bachmann's bundle which reported an association between IAB and atrial fibrosis [14]. 
As a result of the above condition an endothelium dysfunction may be present with a hypercoagulable state comparable to that present in $\mathrm{AF}[15,16]$ and a stagnant and sluggish left atrium favoring the appearance of stasis -induced thrombosis, especially in the left appendage, even in absence of supraventricular arrhythmias.

In the present case, holter EKG monitoring showed $I A B$ and short intermittent episodes of atrial fibrillation. The causes of the different duration of the observed $P$-wave on Holter monitoring may be due to the dynamic registration of the Holter EKG, the semiautomatic measurement and the different day of registration. Moreover, a high CHA2DS2VASc-score was observed (equal to 4) and some studies suggest that patients with high CHA2DS2VASC-score and IAB have a high stroke risk, irrespective of the presence of $\operatorname{AF}[17,18]$. These data support the notion that $A F$ is a risk factor for ischemic stroke, but not necessarily the direct cause of it. On the other hand, in patients with ALS, cardiac arrhythmias are often untreated and symptoms are often attributed to pulmonary or psychiatric cause. But the degree of autonomic dysfunction and IAB may offer insight into the natural course of the disease. Covertible $A F$ is relatively common in patients with ALS. To our knowledge, this is the first reported case of $I A B$ occurring in $A L S$. Due to the high prevalence of $A F$ in patients with $I A B$, the early recognition of advanced IAB by a high CHA2DS2VASC score in ALS patients may help to decide whether antiarrhythmic therapy or oral anticoagulation should be initiated by identifying the patients at a highest risk of AF. Although further randomized clinical trials are warranted in order to formalize such recommendation, IAB may occur in other neurodegenerative illnesses and may shed light into their disease progression as well as potential treatment. Flecainide, a class $1 \mathrm{c}$ antiarrhythmic agent is a strong blocker of persistent late $\mathrm{Na}^{+}$channels in an open conformation. From previous studies using flecanide $[19,20]$ have reported neuroprotective effects without promoting peripheral axonal degeneration but appear to modulate axonal function in ALS patients, stabilizing axonal excitability and reducing a decline in the neurophysiological index. Then, from a previous study, in flecainide treated patients maintained relatively stable peripheral membrane excitability over 32 weeks showing a significant biological activity at the peripheral nerve level [19]. Furthermore, flecainide appears to be effective in maintaining sinus rhythm after paroxysmal atrial fibrillation conversion [21].

\section{Conclusions}

In conclusion, ALS is a demyelinating disease of the brain stem, which sometimes is associated with cardiac conduction abnormalities and arrhythmias. IAB is an atrial conduction disturbance which may induce supraventricular arrhythmias and especially atrial fibrillation. SLA sometimes is associated with atrial arrhythmias especially due to denervation edema. The potential extension of this across Bachman branches may induce an interatrial conduction delay, presenting as IAB. EKG can help to discriminate patients at high risk needing early antiarrhythmic treatment and or potential anticoagulation therapy especially in those with a high CHA2DS2VASc score.

\section{Conflict of Interest}

None.

\section{Sources of Support (If Applicable)}

Disclosure of funding received for this work from any of the following organizations: National Institutes of Health (NIH); Welcome Trust; and other(s) - None.

\section{Statement of Equal Authors' Contribution}

All authors made equal contribution.

\section{References}

1. Pavlovic S, Stevic Z, Milovanovic B, Milicic B, Rakocevic-Stojanovic V, et al. (2010) Impairment of cardiac autonomic control in patients with amyotrophic lateral sclerosis. Amyotroph Lateral Scler 11: 272-276.

2. Shimizu T, Kawata A, Kato S, Hayashi M, Takamoto K, et al. (2000) Autonomic failure in ALS with a novel SOD1 gene mutation. Neurology 54: 1534-1537.

3. Fagius J, Nygren I (2010) Strong potential for baroreflex-governed sympathetic outflow revealed during nausea. Clin Auton Res 20: 371-374.

4. Page RL, Tang AS, Prystowsky EN (1991) Effect of continuous enhanced vagal tone on atrioventricular nodal and sinoatrial nodal function in humans. Circ Res 68: 1614-1620.

5. Rosenbohm A, Schmid B, Buckert D, Rottbauer W, Kassubek J, et al. (2017) Cardiac findings in amyotrophic lateral sclerosis: A magnetic resonance imaging study. Front Neurol 8: 479 .

6. Bayés de Luna A, Cladellas M, Oter R, Torner P, Guindo J, et al. (1988) Interatrial conduction block and retrograde activation of the left atrium and paroxysmal supraventricular tachyarrhythmia. Eur Heart J 9: 1112-1118.

7. Istolahti $\mathrm{T}$, Eranti A, Huhtala H, Lyytikainen L-P, Kahonen $M$, et al. (2020) The prevalence and prognostic significance of interatrial block in the general population. Ann Med 52: 63-73.

8. Spodick DH (2011) Acute pericarditis superimposed on right bundle branch block, posterior fascicular block, and interatrial block. Am Heart Hosp J 9: 112-113.

9. Röcken C, Peters B, Juenemann G, Saeger W, Klein HU, et al. (2002) Atrial amyloidosis: An arrhythmogenic substrate for persistent atrial fibrillation. Circulation 106: 2091-2097.

10. Engelen MA, Juergens KU, Breithardt G, Eckardt L (2005) Interatrial conduction delay and atrioventricular block due to primary cardiac lymphoma. J Cardiovasc Electrophysiol 16: 926.

11. Alexander B, Milden J, Hazim B, Haseeb S, Bayes-Genis A, et al. (2019) New electrocardiographic score for the prediction of atrial fibrillation: The MVP ECG risk score (morphology-voltage-P-wave duration). Ann Noninvasive Electrocardiol 24: e12669. 
12. Martínez-Sellés M (2017) Prevalence and incidence of interatrial block in global population and in different clinical situations. J Geriatr Cardiol 14: 158-160.

13. Asai H, Hirano M, Udaka F, Shimada K, Oda M, et al. (2007) Sympathetic disturbances increase risk of sudden cardiac arrest in sporadic ALS. J Neurol Sci 254: 78-83.

14. Benito EM, De Luna AB, Baranchuk A, Mont L, et al. (2017) Extensive atrial fibrosis assessed by late gadolinium enhancement cardiovascular magnetic resonance associated with advanced interatrial block electrocardiogram pattern. Europace 19: 377.

15. Martínez-Sellés H, Ayesta A, Martínez-Sellés M (2020) Cerebral embolism beyond atrial fibrillation: Interatrial block. CMI 14: 51-59.

16. Larsen BS, Kumarathurai $P$, Falkenberg J, Nielsen OW, Sajadieh A (2015) Excessive atrial ectopy and short atrial runs increase the risk of stroke beyond incident atrial fibrillation. J Am Coll Cardiol 66: 232-241.
17. Bayés de Luna $A$, Martínez-Sellés $M$, Bayés-Genís $A$, Elosua R, Baranchuk A (2017) Surface ECG interatrial block-guided treatment for stroke prevention: Rationale for an attractive hypothesis. BMC Cardiovascular Disorders 17: 211 .

18. Martínez-Sellés $M$, Fernández Lozano I, Baranchuk $A$, Bayes-Genis A, Bayés de Luna A (2016) Should we anticoagulate patients at high risk of atrial fibrillation? Rev Esp Cardiol 69: 374-376.

19. Aliot E, Capucci A, Crijns HJ, Goette A, Tamargo J (2011) Twenty-five years in the making: Flecainide is safe and effective for the management of atrial fibrillation. Europace 13: $161-173$

20. Kanai K, Kuwabara S, Misawa S, Tamura N, Ogawara K, et al. (2006) Altered axonal excitability properties in amyotrophic lateral sclerosis: Impaired potassium channel function related to disease stage. Brain 129: 953-962.

21. Clementy J, Dulhoste MN, Laiter C, Denjoy I, Dos Santos $P$ (1992) Flecainide acetate in the prevention of paroxysmal atrial fibrillation: A nine-month follow-up of more than 500 patients. Am J Cardiol 70: 44A-49A. 